\title{
Germanica
}

\section{Le Voyage en Orient d'Hermann Hesse : un manifeste poétique et politique de l'allégresse}

Die Morgenlandfahrt von Hermann Hesse, ein politisches und poetologisches Manifest der Heiterkeit

Journey to the East from Hermann Hesse, a political and poetical manifesto for cheerfulness

\section{Béatrice Poulain}

\section{OpenEdition \\ Journals}

Édition électronique

URL : http://journals.openedition.org/germanica/5311

DOI : 10.4000/germanica.5311

ISSN : 2107-0784

\section{Éditeur}

Université de Lille

\section{Édition imprimée}

Date de publication : 31 décembre 2018

Pagination : 127-145

ISBN : 978-2-913857-42-1

ISSN : 0984-2632

Référence électronique

Béatrice Poulain, «Le Voyage en Orient d'Hermann Hesse : un manifeste poétique et politique de l'allégresse », Germanica [En ligne], 63 | 2018, mis en ligne le 01 janvier 2021, consulté le 22 janvier 2021. URL : http://journals.openedition.org/germanica/5311 ; DOI : https://doi.org/10.4000/ germanica.5311 


\title{
Le Voyage en Orient d'Hermann Hesse : un manifeste poétique et politique de l'allégresse
}

\author{
Béatrice PoUlAIN \\ Paris Sorbonne, Groupe de Recherche sur la Culture de Weimar
}

Dans Le Voyage en Orient, court récit (Erzählung) rédigé entre 1929 et 1931 et publié en 1932, Hermann Hesse concentre sous forme poétique l'ensemble d'une réflexion sur la langue née lors de la Première Guerre mondiale, face aux débordements haineux d'esprits aveuglés par le nationalisme. La question de la dévalorisation du mot et de la réduction du langage à un système de conventions leurrant l'homme sur la communication véritable qui s'y produit et le détournant de son devenir historique réel, avait plongé Hesse au début des années 20 dans une crise existentielle de production. Dans l'urgence d'un contexte historique où nationalisme et haine se réemparent avec une force destructrice inédite des esprits, Hesse ne sort de cette crise qu'avec la rédaction, fin 1926, début 1927, du Loup des Steppes. Il acquiert alors la certitude que, loin d'être irréversiblement contaminé par ce processus général de perte de sens et de substance, le langage poétique constitue de fait, dans le processus créateur allègre qui le définit, le lieu atemporel de rencontre de l'esprit avec lui-même, là où un jeu authentique avec la langue libère l'esprit d'une aliénation politiquement orchestrée. Cette certitude, ainsi que la conscience historique d'avoir, en sa qualité d'écrivain, pour tâche de la transmettre, sont chez Hesse à l'origine du récit Un Voyage en 
Orient. L'Orient dont il est alors question est éloigné de tout imaginaire exotique de fuite. Il s'attache à la langue poétique comme lieu de naissance (orior) renouvelé et originel de l'esprit à lui-même, comme espace atemporel et essentiellement allègre d'advenir historique de l'humain. Le «Voyage » rapporté par Hesse sous la plume du narrateur H. H., alter ego littéraire dont joue l'auteur, témoignerait de son propre parcours poétique et intellectuel.

La forte dimension autobiographique du récit, étayée par des propos épistolaires de Hesse ${ }^{1}$, apparaît cependant paradoxale au sens où, dans le chapitre final, le narrateur s'efface et disparaît littéralement sous les yeux du lecteur. De même, le récit du « Voyage » se volatilise-t-il, quelques pages plus haut, sous le regard du narrateur sans qu'il ne soit ici ou là question d'allégresse. L'ambivalence et le paradoxe, voire la contradiction, règnent en maîtres dans ce bref récit alors que son enjeu apparaît bien à la fois politique et poétique, si l'on en croit l'auteur face aux recensions de deux critiques contemporains, Heinrich Wiegand et Günter Eich.

Pour son article paru dans Die Neue Rundschau et reconnaissant dans Le Voyage en Orient un " manifeste » politique $^{2}$, Hesse écrit ainsi à Heinrich Wiegand, également collaborateur du Leipziger Volkszeitung et de l'Institut Leipzigeois de Formation des Travailleurs (Leipziger Arbeiter-Bildungsinstitut $)^{3}$ : " Tout ce qui, dans votre essai, se réfère au politique et à l'actualité, me semble excellemment dit, d'une manière claire et pourtant discrète, j'en suis heureux et vous en remercie ${ }^{4}$. » Hesse évoque lui-même son récit comme un «appel au combat» dans d'autres extraits de lettres de la même époque ${ }^{5}$.

La dimension poétique du Voyage apparaît pour le moins aussi cruciale à l'auteur. Quand Günter Eich interroge dans sa recension de

1. - Hermann Hesse, Gesammelte Briefe, vol. 2, Frankfurt a.M., Suhrkamp, 1979, p. 282, «An Alice Leuthold, Mai 1931 » : «Ich habe manchmal mich gefragt, ob ich in dieser Dichtung nicht ein wenig allzu persönlich war und allzuviel ganz Privates mit hineingesteckt habe ».

2. - Heinrich Wiegand, «Hermann Hesses Morgenlandfahrt», Die Neue Rundschau, Fünftes Heft, XLIII. Jahrgang, Berlin und Leipzig, S. Fischer Verlag, Mai 1932, p. 697-701.

3. - Heinrich Wiegand ouvre ainsi pour la création, en 1930, de la pièce brechtienne Grandeur et décadence de la ville de Mahagonny : son engagement et sa lucidité politiques l'amènent à reconnaître dès 1931 que l'Allemagne se situe « sur le bord étroit d'un précipice dévastateur ». Il est contraint dès 1933 à l'exil, en Suisse, où Hesse l'héberge dans un premier temps. Ses écrits sont pour la plupart accessibles dans le recueil d'articles Am schmalen Rand eines wüsten Abgrunds: Gesammelte Publizistik, 1924-1933, éd. par K. Pezold, Leipzig, Lehmstedt Verlag, 2012.

4. - Hermann Hesse, Gesammelte Briefe, op. cit., " Lettre à Heinrich Wiegand, avril $1932 »$, p. 335.

5. - Ibid., novembre 1931, p. 294. 
Kolonne la légitimité d'une écriture qui, s'attachant à « représenter l'irreprésentable », se condamnerait a priori à un échec (missglïcken) dont le livre rendrait témoignage, Hesse le remercie d'avoir su «prendre sérieusement l'auteur au mot et sur le fait », de l'avoir réellement « reconnu », « expérience rarissime » qui « en plusieurs décennies, ne s'est produite que très peu de fois ${ }^{6}$.

De fait, Le Voyage en Orient semble réellement constituer un échec dans le sens où son propos même de manifeste échappe entièrement, sur le double plan politique et poétique, à la réception actuelle. KlausPeter Philippi reproche ainsi à l'auteur du Voyage en Orient de n'avoir participé à aucun des " débats esthétiques et poétologiques » de son siècle « sur les formes et fonctions de la littérature » et d'avoir opté pour un escapisme, une «mentalité de fuite » et une « occultation de la politique » à l'origine des ventes aujourd'hui encore hors norme de ses ouvrages en librairie. Si son penchant au « surréalisme » conduit Hesse à évoquer une forme de «magie ancestrale » dans Le Voyage en Orient, cette dernière se muerait sous sa plume en un « tour de passepasse » d'une fabrication de légendes selon des motifs religieux dont « le programme consiste à s'éloigner de la réalité de la vie pour entrer dans un monde dématérialisé de l'esprit et de l'art »7.

L'ambivalence et la bipolarité du récit de Hesse furent en revanche tellement appréciées d'André Gide qu'il demanda à son gendre de traduire Le Voyage en Orient en français. L'introduction dont il dote alors la version française reproduit l'ambivalence caractérisant l'ouvrage, puisqu'elle comporte deux parties, la première soulignant l'engagement et la clairvoyance politiques de l'auteur, la seconde sa pensée poétique dans son souci de vérité et son ancrage culturel :

L'œuvre entière de Hesse est un effort poétique d'émancipation en vue d'échapper au factice et de réassumer l'authenticité compromise. Avant de l'enseigner au-dehors, il importe de la préserver en soi. Hesse y parvient par la culture ${ }^{8}$.

S'y ajoute un troisième point, inédit, que Gide estime tout particulièrement, « l'ironie latente » qui traverse tout le récit. Le propos de Gide

6. - Hermann Hesse, « Briefe », Gesammelte Werke, Bd. 7, Betrachtungen und Briefe, p. 522 : «Es ist außerordentlich selten, daß ein Autor durch eine Kritik ernstlich angefaßt und gestellt wird, mir ist es in mehreren Jahrzehnten nur ganz wenige Male geschehen ».

7. - Karl-Peter Philippi, « HESSE und die heutige Germanistik in Deutschland », in : Jahrbuch der Internationalen Hesse-Gesellschaft, Tübingen, Niemeyer, 2003, p. 10.

8. - André Gide, Préface au Voyage en Orient, traduit de l'allemand par Jean Lambert, Paris, Calmann-Lévy, 1948 ; ici Paris, Librairie Générale Française, le Livre de Poche, 2007, p. 7-14. 
reste cependant éclaté, les pôles politique et poétique disjoints, l'ironie constatée un troisième fragment de lecture.

Sur le plan formel, l'ironie représente en tout état de cause l'expression stylistique de l'ambivalence et, dans sa réponse à Eich, Hesse souligne que ce dernier a parfaitement reconnu dans « le paradoxe (mieux : la bi-polarité) » le « sens intime » de l'œuvre, là où elle se laisse le mieux saisir (fassen $)^{9}$. On retrouve ici l'ambivalence de la réception exprimée sur un mode programmatique. L'hypothèse de lecture sera ainsi la suivante : seul un regard considérant l'allégresse, et avec elle l'ironie et l'humour, comme point nodal de l'écriture, permettra de saisir Le Voyage en Orient dans son ensemble, dans le jeu assumé de tous ses fragments.

Dans cette perspective, Le Voyage en Orient constitue un manifeste à la fois poétique et politique dont le caractère ambigu, insaisissable représente l'expression formelle recherchée. L'« échec » orchestré et apparent, pour Günter Eich comme pour Maurice Blanchot ${ }^{10}$, d'une écriture poétique achoppant à l'écueil de «l'allégorie », en réaliserait de fait allègrement l'accomplissement évidé, " non agressif », « en apparence inoffensif $»^{11}$. Il n'étonnera alors plus que ce manifeste pour une poétique de l'allégresse ne recoure lui-même au terme de Heiterkeit qu'exceptionnellement, mais à des moments-clefs, dans un texte qui s'écrit aussi par son silence.

Le manifeste politique repéré par Heinrich Wiegand dans Le Voyage en Orient et analysé ici dans un premier temps constitue alors l'envers ironique d'un manifeste poétique de l'allégresse qui, deuxième temps d'analyse, fonde sa réflexion poétologique sur l'impossibilité poétique et la nécessité politique d'une «narration historique » véridique et recourt pour ce faire formellement à l'allégorie.

\section{Le Voyage en Orient : un « appel au combat », un « manifeste » politique}

Ainsi que le souligne Hesse dans sa lettre à Alfred Kubin, Le Voyage en Orient semble aux yeux non avertis bien éloigné de l'actua-

9. - H. Hesse, «Briefe », Gesammelte Werke, Bd. 7, op. cit., p. 522.

10. - Maurice Blanchot, Le Livre à venir, « H. H. », Paris, Gallimard coll. Folio, p. 227-251. Ici p. 240 : «Manifestement, ce que Hesse souhaite, c'est unir la féérie et l'allégorie [...]. Mais, de même que le voyage s'enlise dans les contestations et les doutes, de même dans le récit la naïveté se subtilise au contact de l'allégorie, et l'allégorie devient naïveté ».

11. - H. Hesse, Gesammelte Briefe, op. cit., Lettre à Alfred Kubin, fin mars 1932, p. 329 : «Die Morgenlandfahrt stößt auf mehr Verständnis als ich erwartet hatte, wahrscheinlich [...] weil dies Buch scheinbar harmlos und freundlich bleibt und gar nichts Aggressives hat ». 
lité et des préoccupations politiques de ses contemporains. L'auteur qualifie d'ailleurs lui-même volontiers ce « récit » (Erzählung) de « conte» (Märchen $)^{12}$, ce qui s'explique par ses dimensions « féerique » et « surréaliste » $\left(\right.$ Blanchot) ${ }^{13}$. D'entrée de jeu, le voyage « extraordinaire » (unerhört) dont est ici tentée la narration, se place en effet, selon le narrateur H. H., dans la lignée du Roland Furieux et de son merveilleux sur fond d'amour et de guerre, d'Eros et de Thanatos, aux confins de la magie et du rêve ${ }^{14}$. Guidé par son amour pour la princesse Fatma, ce voyage est entrepris par H. H. dans sa jeunesse, «dans un monde ébranlé par la Première Guerre mondiale » (ML 29), alors que la «pensée »(Denken) se voit, notamment chez « les peuples vaincus », confrontée à « un extraordinaire état d'irréalité » et s'ouvre à « la surréalité » (Bereitschaft zum Überwirklichen) (ML 9). Son périple le conduit avec ses compagnons, membres de l'« Ordre » secret et séculaire des « Pèlerins de l'Orient", à réaliser de fait des « percées dans un monde héroïque et magique » (ML9) : les Pèlerins traversent non seulement des « espaces » réels et légendaires tels la Souabe et « la Mer de la Lune » mais aussi «les temps » (Zeiten), le Moyen Âge et l'Âge d'Or (ML27). Ils rencontrent aussi des hauts personnages de l'Ordre historiques et fictifs, tels Lao Tse, Platon, Hoffmann, Don Quichotte, Witiko ou Goldmund (ML33). C'est que l'espace-temps du Voyage est celui de l'esprit : sa destination n'est ni un lieu, ni un temps précis. Ce voyage est sans âge et mène de tout temps les « esprits » (Geister, ML16) au " Royaume des Contes » (Märchenreich) (ML14), aussi «patrie de la lumière » (Heimat des Lichts, ML15), " patrie et jeunesse de l'âme » (Heimat und Jugend der Seele), « synthèse de tous les temps » (Einswerden der Zeiten) (ML28).

La parenté est manifeste avec le rêve romantique de "poétisation » du monde et de l'homme de Novalis' 15 , cité comme l'Arioste en qualité de parrain dès les premières pages du récit ${ }^{16}$. Cependant - Heinrich Wiegand le souligne dans sa recension ${ }^{17}$ - les allusions ironiques de

12. - Cf. par exemple H. Hesse, Gesammelte Briefe, op. cit., novembre 1931, p. 291.

13. - Cf. M. Blanchot, Le Livre à venir, op. cit., p. 240, et H. Hesse, Gesammelte Briefe, op. cit., «Lettre à Albrecht Goes, janvier 1948 », p 462 : « Sollte ich je in diesem Leben noch etwas dichten, so würde es vermutlich noch surrealistischer sein als die Morgenlandfahrt und ähnliche Sachen ».

14. - Hermann Hesse, Die Morgenlandfahrt, Frankfurt a.M., Suhrkamp, 1982, p. 7. Dorénavant cité dans le texte, sigle ML.

15. - Cf. Jürgen Söring, « Über die Grenze der Darstellbarkeit. Novalis und Hesse », Jahrbuch der deutschen Schillergesellschaft, Berlin, De Gruyter, 1977, p. 468516.

16. - H. Hesse, Die Morgenlandfahrt, op. cit., p. 16: « ... das Wort des Dichters Novalis : "Wo gehen wir denn hin? Immer nach Hause" ».

17. - H. Wiegand, «Morgenlandfahrt », op. cit, p. 699 : «Die oberflächliche 
Hesse dans le texte lui permettent non seulement de spécifier que ce romantisme n'a rien de passéiste ni de réactionnaire mais se dresse au contraire contre toute réécriture nationaliste et falsificatrice de l'histoire à la fois politique et artistique de l'Allemagne.

Son narrateur H. H. relate ainsi plusieurs rencontres désagréables avec les «Gardiens de la Couronne » (Kronenwächter) d'Achim von Arnim qui cherchent sans succès à gagner à leur cause le mouvement des Pèlerins de l'Orient (ML19) : toute nostalgie d'Empire, voire de « Troisième Reich » (ML13), tout monarchisme au nationalisme antinapoléonien, donc anti-français, sont rejetés avec fermeté par les dirigeants de l'Ordre - comme par l'auteur. Il convient en effet de souligner que Hesse se compte lui-même au nombre des Pèlerins de l'Orient, « la seule communauté à laquelle il eût jamais appartenu $»^{18}$.

À travers Les Gardiens de la Couronne et la voix de son narrateur, Hesse critique également l'écriture contemporaine de l'histoire, marquée par de «nombreux romans et faux romans (Halbromane) historiques et biographiques » peu soucieux de « recherche "exacte" » ou de vérité historique ${ }^{19}$. Ce discrédit des sciences historiques se retrouve dans le traitement politique du passé le plus récent, dans la réalité de Weimar et sa transcription fictive. Lorsque H. H. dénonce dans « le désir d'oubli » « le désir le plus violent et le plus aveugle de l'homme » (Sehnsucht, ML11), c'est pour déplorer que « des peuples entiers » en soient venus à « oublier, nier, supprimer, magiquement effacer » « une guerre monstrueuse, horrible, qui a duré pendant des années ». L'Allemagne préhitlérienne où gronde, Hesse l'écrit dans une lettre, la « menace d'un terrorisme fasciste ${ }^{20}$ est manifestement visée par l'auteur. En 1932, année de parution, rappelons-le, du Voyage en Orient, l'une des lettres retenues personnellement par Hesse dans le septième volume de ses Cuvres Complètes (1957) est plus explicite encore, car l'auteur s'y adresse indirectement à la jeunesse. Son destinataire, Arno Steglich, directeur d'une auberge de jeunesse allemande (DJH) qui sera interdite dès 1933, en communiquera en effet des extraits «pertinents » (geeignet) «à ses jeunes amis »:

Gleichsetzung von romantischem Wissen mit Neigung zum Rückschritt wird überdies vom Erzähler selber ironisiert... ».

18. - H. Hesse, Gesammelte Schriften VII, op. cit., « Brief an eine Leserin des Glasperlenspiels, September 1947 », p. 666 : «Ich selbst habe [...] nie irgend einer Gemeinschaft angehört, außer jener der Morgenlandfahrer, eines Bundes von Gläubigen, dessen Existenzform eine sehr ähnliche ist wie die Kastaliens ».

19. - H. Hesse, Gesammelte Briefe, vol. 2, op. cit., Article du 2.10 .1935 dans le National-Zeitung de Bâle, p. 480.

20. - H. Hesse, Gesammelte Briefe, vol. 3, op. cit., Brief an Ludwig Finckh, Februar 1933, p. 315. 
... ainsi je ressens de l'aversion et de l'hostilité surtout pour la forme allemande actuelle du nationalisme. L'effacement mensonger de toute responsabilité de la guerre, l'imputation de l'état de l'Allemagne aux seuls « ennemis » et à Versailles, génère selon moi une atmosphère de bêtise politique, d'hypocrisie et d'immaturité en Allemagne qui va fortement contribuer à la naissance d'une guerre future ${ }^{21}$.

La «falsification » nationale-socialiste « de l'histoire » prend selon Hesse sa source dans cette « virtuosité allemande à l'effacement mensonger (Weglügen) et à l'oubli (Vergessen) de ses propres péchés »22, notamment de sa responsabilité dans le déclenchement de la Première Guerre mondiale. Elle va de pair avec un vitalisme pseudo-nietzschéen se défiant des sciences historiques et de l'esprit en général. Cette « révolte vigoureuse de notre vitalisme » (Lebensgefühl), ce « renoncement » à l'histoire sont cependant loin de nous « rendre notre innocence », écrit Hesse dans un essai de 1930, Magie du Livre. Une telle défiance est au contraire fatale car c'est dans sa conscience historique d'elle-même que se fonde l'humanité23. Ces propos contrent une lecture abusive de Nietzsche en transmettant déjà la pensée burckhardtienne de l'histoire, avant son immortalisation littéraire sous les traits du Père Jacobus dans Le Jeu des perles de verre. Dans ses Considérations sur l'histoire universelle, Jacob Burckhardt souligne en effet que, dépossédée de son histoire, l'humanité sombre dans la «barbarie » de la pure « matérialité » (des Stoffes). Or le cœur de cette histoire, son moteur invariant, « changeant mais impérissable », c'est l'esprit (Geist). L'esprit constitue selon Burckhardt le principe dynamique générant sans cesse bouleversements et relatifs équilibres des trois «puissances » (Potenzen) historiques que sont l'État, la religion et la culture ${ }^{24}$. Il relève alors du

21. - H. Hesse, Gesammelte Schriften, vol. 7, op. cit., Brief an Herrn Arno Steglich, etwa 1932, p. 539 : « ... so fühle ich besonders für die jetzige deutsche Form von Nationalismus eine Abneigung und Feindschaft. Das Weglügen aller Kriegsschuld, das Verschieben aller Verantwortung für Deutschlands Zustände auf die "Feinde" und auf Versailles, erzeugt nach meiner Auffassung in Deutschland eine Luft von politischer Dummheit, Verlogenheit und Unreife, die zur Entstehung eines künftigen Krieges sehr viel beitragen wird ».

22. - H. Hesse, Gesammelte Briefe, vol. 3, op. cit., Lettre à Otto Hartmann, février 1935, p. 455 ; puis Hermann Hesse, Politik des Gewissens, vol. 2, Frankfurt a. M., Suhrkamp, 1981, Lettre de novembre 1932 à Erhard Bruder, p. 497.

23. - H. Hesse, Gesammelte Schriften, vol. 7, op. cit, « Magie des Buches », p. 343-354 : «Ohne Wort, ohne Schrift und Bücher gibt es keine Geschichte, gibt es nicht den Begriff der Menschheit. [...] Es wird sich zeigen, daß die Formulierung durch das Wort und die Überlieferung dieser Formulierungen durch die Schrift [...] die einzigen Mittel sind, kraft dessen die Menschheit eine Geschichte und ein fortdauerndes Bewußtsein ihrer selbst haben kann ».

24. - Cf. Jacob Burckhardt, Über das Studium der Geschichte, éd. par Peter Ganz, München, C.H. Beck, 1982, p. 15 et 228. 
devoir de l'historien de « conserver et de préserver le plus haut bien » de l'humanité toute entière, «la continuité de l'esprit créateur de culture » - la culture représentant l'objectivation ou la concrétisation de l'esprit humain ${ }^{25}$.

Tel est aussi l'objectif poursuivi par Hesse dans Le Voyage en Orient, lequel propose de fait un parcours spatio-temporel inédit à travers l'ensemble de la culture humaine, non seulement occidentale, encore moins spécifiquement allemande, mais aussi indienne, chinoise, arabe. Contre toute forme de nationalisme, Hesse y propose simultanément un contreexemple aux « formes de communauté très primitives et hostiles à l'esprit » qu'offrent à ses yeux le communisme et le fascisme ${ }^{26}$. «L'Ordre » représente une communauté intemporelle de l'esprit dont les membres les plus accomplis, les plus imprégnés de culture et d'esprit, les plus croyants aussi, se distinguent par leur allégresse (Heiterkeit, ML 19, ML 59) au même titre que leurs prédécesseurs, les «Immortels » du Loup des Steppes, ou que leurs successeurs, les « Joueurs des perles de verre ». Cette allégresse est militante, œuvre contre la barbarie, à l'image de ce que recommandait aussi Burckhardt, cité presque mot pour mot par le Père Jacobus dans Le Jeu des perles de verre:

Des périodes de terreur et de très profonde misère peuvent survenir. Mais s'il doit y avoir encore un bonheur dans la misère, ce ne peut être qu'un bonheur de l'esprit, orienté, dans le passé, vers le sauvetage de la culture des époques antérieures, et, pour l'avenir, vers l'affirmation [allègre] et persévérante de l'esprit, dans une ère qui sans cela risquerait d'être entièrement vouée à la matière 27.

Dans Le Voyage en Orient, Hesse oppose ainsi une réalité à une autre. Si la réalité vécue par la culture, au sein d'une communauté atemporelle de l'esprit, fait classiquement front à « la morne brousse de la banalité quotidienne » et des affaires (ML31), cette dernière est simultanément

25. - Helmut Schnädelbach, Geschichtsphilosophie nach Hegel. Die Probleme des Historismus, Freiburg, München, Alber Kolleg Philosophie, 1974, p. 74.

26. - H., Hesse, Gesammelte Briefe, op. cit., « À G. Rutishauser, décembre 1935 », p. 489.

27. - Hermann Hesse, Das Glasperlenspiel, Frankfurt a. M., Suhrkamp, 1972, p. 400 : «Es können Zeiten des Schreckens und tiefsten Elends kommen. Wenn aber beim Elend noch ein Glück sein soll, so kann es nur ein geistiges sein, rückwärts gewandt zur Rettung der Bildung früherer Zeit, vorwärts gewandt zur heitern und unverdrossenen Vertretung des Geistes in einer Zeit, die sonst gänzlich dem Stoff anheim fallen könnte. » Version française, Le Jeu des perles de verre [1955], trad. J. Martin, Paris, Livre de poche, 2008, p. 476. Les propos authentiques de Burckhardt, issus de ses Vorlesungen über die Geschichte des Revolutionszeitalters (p. 19), sont majeurs au point d'être cités par Peter Ganz dans son introduction à l'édition de la version intégrale des «Considérations sur l'histoire universelle ». Cf. J. Burckhardt, Über das Studium der Geschichte, op. cit., p. 71. 
celle qu'il dénonce dans ses lettres et articles, une réalité de « la guerre, la technique, l'ivresse de l'argent et du nationalisme », ces «idoles actuelles » (Zeitgötzen) « à la place desquelles il faut pouvoir placer une foi (einen Glauben setzen) $\gg^{28}$. La réalité visée concerne particulièrement l'Allemagne : dès 1927, Hesse dit « ignorer en combien d'autres pays la haine d'un peuple entier envers tout ce qui relève de l'esprit (alles Geistige) est aussi unanime et quasi-organisée qu'en Allemagne ${ }^{29}$.

Selon Hesse, sa propre tâche consiste à « opposer d'abord au chaos l'esprit » puis à « transmettre aux générations futures » « la foi en l'esprit comme créateur et comme logos $»^{30}$. Cette foi est aussi celle d'Harry Haller dans les «Immortels » « allègres » du Loup des Steppes, ou de H. H. dans les « Pèlerins » et leur allégresse ${ }^{31}$. Dans son essai de 1932, Dank an Goethe, Hesse précise qu'il entend, comme Goethe, par esprit « la vérité et servir la vérité », ce qui interdit tout nationalisme, toute « écrivasserie mensongère (verlogene Schreiberei) et primitive » et oblige à réfléchir au problème le plus brûlant pour Goethe comme pour l'époque, à celui d'un " gouvernement (Regierung) de la vie humaine par l'esprit ${ }^{32}$. La foi que Hesse exprime «par images poétiques » (dichterisch) dans Le Voyage en Orient, et « directement » dans son poème Besinnung de novembre 1933, est « le contraire de la religion du sang et du pouvoir $\gg$ (Blut- und Machtreligion) alors aux commandes ${ }^{33}$. L'objectif de Hesse est dans les deux cas de prendre explicitement position pour une vision « logocentrique » et non « biocentrique » de l'histoire (Anschauungsart) ${ }^{34}$. Le Voyage en Orient relève donc bien, comme le soulignait Heinrich Wiegand dans sa recension, d'un «manifeste » où Hesse « combat » « l'infâme trahison d'un règne de l'esprit » ainsi que le discrédit jeté à la fois sur « la formation de l'esprit » (geistige Bildung) et

28. - H. Hesse, Gesammelte Schriften, VII, op. cit., « Brief an Herrn B., etwa $1930 »$, p. 501.

29. - Cf. « Lettre de juillet 1927 à Hilde Jung-Neugeboren » in : Materialien zu Hermann Hesses Steppenwolf, éd. V. Michels, Frankfurt a.M., Suhrkamp, 1972, p. 122.

30. - Hermann Hesse, Politik des Gewissens, vol. II, « Aus einem Brief vom Mai 1934 an Friedrich Michael », Frankfurt a. M., Suhrkamp,1982, p. 553 : « Nicht [den Todeskampf] zu verhindern, zu verzögern oder zu beschleunigen halte ich für meine und unsere Aufgabe, sondern ihn zu sehen, den Blick ins Chaos zu ertragen, dem Chaos den Geist entgegenzustellen und den Glauben an den Geist, als Creator wie als Logos, den Späteren weiterzugeben ».

31. - Cf. H. Hesse, Gesammelte Briefe, vol. 2, op. cit., « Lettre de janvier 1933 », p. 363 .

32. - Hermann Hesse, Dank an Goethe, Zürich, Werner Classen Verlag, 1946, p. 7-19, ici p. 14.

33. - H. Hesse, Politik des Gewissens, op. cit., «Lettre du 1.1. 1934 à Arthur Stoll», p. 547.

34. - Hermann Hesse, Schriften zur Literatur I, « Brief, 1935 », Frankfurt a. M., Suhrkamp, 1970, p. 99. 
la « littérature » (Dichtung), et où il dénonce la tentative nationale-socialiste de « briser les passerelles menant aux meilleures traditions intellectuelles ». Rappelons que Hesse se réjouit explicitement dans une lettre de 1931 que ses lecteurs, "plutôt jeunes et modernes » " voient dans ce conte, comme d'ailleurs dans toutes [ses] œuvres, non un "romantisme" et une fuite devant la vie, mais au contraire des appels au combat, des exhortations à la persévérance et à la fidélité (Treubleiben) » ${ }^{35}$.

La posture historique héritée de Burckhardt qui lui fait rejeter toute incursion de l'actualité dans l'art et l'écriture ${ }^{36}$ pour mieux consacrer cette dernière à l'évocation d'une réalité d'art et de culture traversée de beauté et d'allégresse, pourrait cependant receler le piège d'un dire affirmateur, d'une positivité, récupérables par les forces politiques mêmes que Hesse combat. L'auteur en est conscient, lui-même honnit «l'optimisme du bourgeois » et les attentes «bourgeoises » d'un art « affirmant la vie au moyen du mot $»^{37}$ :

Le principe voulant que la littérature existe afin de proposer au peuple une nourriture simple, saine, réjouissante (erheiternd) et aidant à surmonter les conflits, ce principe, Monsieur Goebbels ou le Général Franco le partageront sans conteste avec vous, mot pour $\operatorname{mot}^{38}$.

À partir du Loup des Steppes, la pensée poétologique de Hesse lui permet d'éviter l'écueil de l'embellissement mensonger vilipendé par Nietzsche dans l'art apollinien ${ }^{39}$ et reproché à Goethe par Harry Haller lors de leur face-à-face du " Théâtre magique », préfiguration de l'espace-temps spirituel et culturel du Voyage en Orient. L'urgence politique amène en effet Hesse à élaborer une poétique de l'allégresse aux accents dionysiaques et pourtant non nietzschéenne qu'il thématise simultanément à sa mise en œuvre dans tous ses écrits. Le Voyage en Orient en constitue le manifeste.

35. - H. Hesse, Gesammelte Briefe, vol. 2, op. cit., « Lettre à Helene Welti, novembre $1931 »$, p. 294.

36. - H. Hesse, Politik des Gewissens, op. cit., " Lettre de septembre 1933 à Werner Reinhart », p. 543.

37. - H. Hesse, Gesammelte Briefe, vol. 2, op. cit., Lettres de 1926 et 1927, p. 140 et 172 .

38. - Materialien zum Steppenwolf, op. cit., «Brief, Jan/Feb. 1937 », p. 151 : « Den Grundsatz, daß die Dichtung dazu da sei, dem Volk einfache, gesunde, erheiternde, über Konflikte weghelfende Kost zu bieten, diesen Grundsatz wird ohne Zweifel Herr Goebbels oder General Franco wörtlich mit Ihnen teilen ».

39. - Cf. Friedrich Nietzsche, Die Geburt der Tragödie, Kritische Studienausgabe, tome 1 (KSA 1), éd. par G. Colli und M. Montinari, München, Berlin, New York, dtv/ de Gruyter, 1988. 


\section{Le Voyage en Orient : un manifeste allégorique de la poétique de l'allégresse hesséenne}

Dans tous ses écrits postérieurs à la Première Guerre mondiale, la Heiterkeit est thématisée par Hesse de manière nietzschéenne en ce que son caractère idéal n'est plus entamé par un esprit de la modernité vécu comme fatalité : l'harmonie grecque naïve au fondement de l'idéal d'allégresse classique, notamment schillérien, s'enrichit d'une dissonance tragique tout aussi grecque qui n'est plus à surmonter à l'instar de la fissure mélancolique sentimentale, symptôme de modernité ${ }^{40}$, mais au contraire à accueillir et à intégrer pour tout être et tout art visant son accomplissement dans l'idéal d'allégresse.

Hesse thématise ainsi toujours la Heiterkeit sur fond de crise essentiellement existentielle. Elle constitue l'apanage d'un être supérieur car en harmonie, par l'esprit et l'art, avec lui-même, autrui et le monde, tels le magicien Jup du Dernier été de Klingsor (1919), Vasudeva dans Siddhartha, " les Immortels » Goethe ou Mozart dans Le Loup des Steppes, ou encore les supérieurs hiérarchiques de l'Ordre des Pèlerins dans Le Voyage en Orient. L'allure «élastique et allègre » (ML59) de Léo notamment correspond à sa faculté à " vivre sans cesse dans une intimité palpitante avec son entourage » (immerzu in fließender, wogender Beziehung und Gemeinschaft mit seiner Umgebung zu stehen, ML 66). Cette Heiterkeit apparaît telle une image de rêve, un idéal de bonheur et de communion de l'un avec le tout ${ }^{41}$, à un personnage principal marqué au contraire, tel Harry Haller dans Le Loup des Steppes ou H. H. dans Le Voyage en Orient par la souffrance, la solitude, la maladie, la mélancolie, le désespoir. La mort constitue ainsi le cœur des cinq chapitres du Voyage en Orient dont les trois chapitres centraux sont consacrés au chaos, au vide, à l'oubli, sous la double thématique de la Grande Guerre et de l'impossibilité de toute écriture d'histoire.

Hesse s'avère en cela plus fidèle au Nietzsche de La Naissance de la tragédie, ouvrage rédigé spécifiquement contre l'allégresse grecque schillérienne, que Nietzsche lui-même dans ses derniers écrits. Mû par la nécessité d'une justification par les œuvres, Nietzsche adhère à son insu à l'attitude schillérienne de l'essai Sur Bürger, quand l'œuvre allègre finit par ne pouvoir être à ses yeux que le produit d'une grande santé, d'une force active saine et allègre. Dans son orchestration de jeux

40. - Cf. Friedrich Schiller, Über naive und sentimentalische Dichtung [1795], p. 407-582, in Werke V:Philosophische Schriften - Vermischte Schriften, éd. par Helmut Koopmann, München, Winkler, 1975.

41. - Cf. H. Hesse, « Glück» in : Glück. Späte Prosa, Frankfurt a.M., Suhrkamp, 1974, p. 42-56, ici p. 49 : « Atmen in vollkommener Gegenwart, Mitsingen im Chor des Sphären, Mittanzen im Reigen der Welt, Mitlachen im ewigen Lachen Gottes, das ist unsre Teilhabe am Glück ». 
de masques, il opte pour la positivation dans Ecce Homo où il en vient, « dieu » promenant son oisiveté le long du Pô, à arborer une allégresse divine dépourvue de toute tension tragique ${ }^{42}$. S'il est bien question d'une « psychocratie » dans Le Voyage en Orient et d'« Immortels » au « rire surhumain » dans Le Loup des Steppes, Hesse souligne qu'il s'agit toujours d'une communauté de «Serviteurs aimants » (die Liebenden und Dienenden $)^{43}$ se dédiant à l'esprit comme vérité : le pouvoir, « mauvais en soi » selon Burckhardt, rend «malades » ceux qui le convoitent à des fins matérielles, égoïstes, non dictées par l'esprit, explique Léo dans Le Voyage en Orient (ML34). Ce plus haut dignitaire de l'Ordre incarne au contraire « le serviteur idéal » (ML26) de l'esprit et de la culture humaine universelle. Porteur de semelles de corde et habitant au Seilergraben (ML60), cette variante infidèle du funambule nietzschéen entend préserver et non détruire, tel le lion de la parabole zarathoustrienne, une culture dont il représente la quintessence. Il se distingue par sa faculté d'apparition et de disparition (ML26, 35) et une humilité lui permettant de se fondre, inoffensif (harmlos) et insaisissable, dans tout environnement. Une instrumentation idéologique telle que Nietzsche en était alors l'objet dans l'Allemagne préfasciste, devient ici impossible. Tel est bien l'un des enjeux hesséens dans son écriture de l'allégresse : se prémunir du double écueil nietzschéen et classique d'une auto-idéalisation solaire et olympienne du génie créateur qui pourrait assimiler ce dernier à un Führer. Rien n'est en effet plus « abject » (verhasst) aux yeux de Hesse au début des années 30, ainsi qu'en témoignent les lettres choisies des Euvres complètes ${ }^{44}$. La proximité entre le narrateur $\mathrm{H}$. H. et l'auteur Hesse s'en trouve confirmée :

Ce que vous exigez du poète dans votre lettre ouverte en convoquant le Goethe « olympien » à l'appui, cette vue d'en haut (Darüberstehen) olympienne et détachée, ce n'est là précisément pas ma tâche (Aufgabe). [...] je ne ressens en rien comme tâche de masquer ou de présenter comme bénins les abîmes de la vie humaine en générale et de la mienne en particulier, mais au contraire de reconnaître, d'exprimer et de partager la souffrance et les tourments de la condition humaine précisément sous leurs formes actuelles. [...] Je confesse réellement abhorrer le mot de Führer dont la jeunesse allemande abuse tant. Ne nécessite et n'exige un Führer que celui qui ne souhaite aucune responsabilité, aucune pensée propres ${ }^{45}$.

42. - F. Nietzsche, KSA 6, Ecce Homo, « Götzen-Dämmerung », op. cit., p. 356.

43. - H. Hesse, Gesammelte Schriften, vol. 7, op. cit., p. 543.

44. - Ibid., Lettres de 1931, 1932, 1933, p. 513, 517, 529, 540, 563 etc.

45. - Ibid., « Lettre au Dr. M.A. Jordan, 1932, Réponse à la lettre ouverte "La mission du poète" », p. 532. 
La passivité de la souffrance et de la maladie, la «persévérance dans le chaos » (Ausharren inmitten des Chaos) sont dans cette lettre opposées à tout activisme, tout volontarisme d'un Führer arborant « une supériorité et une santé imperturbables » (Unangefochtenheit). C'est l'attitude même du narrateur $\mathrm{H}$. $\mathrm{H}$. face à l'écriture de son récit de Voyage en Orient : mélancolique (trüb), malade, désespéré, il entend ne pas baisser les bras «face au chaos » et persévérer dans « son entreprise en apparence impossible » (ML46). De même, Hesse souligne fréquemment dans ses lettres combien son attitude paraît « ridicule » (lächerlich), « aberrante » (töricht), « donquichottesque » au grand nombre, quand le seul « héroïsme » acceptable pour « la confrérie anonyme » des « serviteurs » de l'esprit et de la vérité est de mourir, non de «faire mourir » pour sa cause $\mathrm{e}^{46}$.

Dans Le Voyage en Orient, " l'Ordre des Pèlerins », généralement perçu comme " cortège de bouffons » ou " croisade des enfants » (ML11,18), relève du même ridicule prêtant à sourire ironiquement (ML47,49), ce qui n'empêche pas le narrateur H. H. de lui offrir, héroïquement, sa vie. Il parachève le destin réservé aux écrivains membres de l'Ordre qui, «pâles », « à demi-réels », manquant " d'authenticité » $(e c h t)$, sont loin d'être célébrés pour leur puissance créatrice, tandis que leurs personnages apparaissent "plus beaux, plus gais (froher), plus justes, plus réels » (ML33). La cause en est, selon Léo, incarnation de la « sagesse » de l'Ordre, le processus d'écriture lui-même qui, ressortant de « la loi du servir» (Gesetz des Dienens) au fondement de l'Ordre, est don des forces vives de l'auteur à ses créatures fictives (ML34). Il est surtout offrande sacrificielle du sujet pensant et écrivant à la vérité de connaissance, comme en témoigne le devenir du narrateur H. H. qui, au cours de son récit du Voyage, comme au terme de la nouvelle Le Voyage en Orient, perd de plus en plus sa réalité de sujet, jusqu'au néant, jusqu'à se laisser fondre totalement en Léo, au cœur d'une statuette cultuelle « incompréhensible » qui, réunissant dos à dos les images des deux personnages, lui apporte ce faisant la connaissance de soi recherchée (ML101).

Telle est en effet l'épreuve finale, celle de la connaissance de soi, à laquelle l'Ordre condamne $\mathrm{H}$. $\mathrm{H}$. lorsque ce dernier passe devant son tribunal pour l'avoir délaissé : il lui faut lire l'archive de l'Ordre, dépositaire de vérité, sur lui-même. Le périple, doublement raté, du « Voyage en Orient » effectif de H. H., dans ses jeunes années, durant lequel l'Ordre s'est perdu à ses yeux, comme du récit de cette aventure où H. H. souhaitait écrire l'histoire de son Voyage et de l'Ordre, s'achève donc sur la disparition définitive du narrateur mélancolique comme

46. - Ibid., « Lettre à un jeune homme en Allemagne, 8 avril 1932 », p. 518. 
vérité poétique. Aucun narrateur souffrant de la modernité n'apparaîtra d'ailleurs plus dans les écrits de Hesse : Le Jeu des perles de verre est entièrement consacré au successeur de Léo, au Maître du Jeu Josef Knecht, et le narrateur y est un historien castalien relevant de l'allégresse caractérisant toute la " Province Pédagogique ». C'est que Le Voyage en Orient réalise sur un mode poétologique une poétique de l'allégresse à laquelle Hesse va dorénavant lui-même dédier ses forces en qualité d'historien-passeur d'une réalité et d'une communauté culturelle, objective et actuelle.

Cette confession de foi relève de la dimension autobiographique du Voyage mais n'a jusqu'à présent pas été identifiée : l'auteur ne la donne humoristiquement à lire que de manière cryptée, par jeu de pistes. En effet, l'infidélité à l'Ordre qui apparaît sur l'étiquette de l'archive H. H., « Chattorum r. gest. XC./civ. Calv. infid. 49 » (ML95) correspond à sa propre épreuve de perte de foi en l'esprit, ou plus précisément en l'écriture, en la langue poétique : en 1926, à 49 ans, et avant la «catharsis » 47 du Loup des Steppes, Hesse, ancien « citoyen de Calw », descendant des "Chatti », tribu germanique du Land de Hesse, était en pleine crise. Ainsi qu'il l'écrivit dans une lettre, son travail d'intellectuel et d'écrivain lui paraissait pure « illusion », " dénué de toute valeur » car participant de l'effroyable « dévalorisation de la langue et du mot » en cette époque de grand « délabrement intellectuel » (Zerfall der Geistigkeit) : « Nous pourrions tout aussi bien faire de simples bulles de savon ${ }^{48}$. Au plus profond de la crise et de l'échec, au cœur du chaos et de la vacuité de sens, Hesse trouve son salut existentiel et poétique dans l'humour et l'allégresse. Les «bulles de savon » préfigurent en effet les " perles de verre », symbole d'un art achevé, d'un art allégorique jouant avec le vide, et dans son entreprise poétique de vérité, Hesse s'emploie dorénavant à une «justification de l'illusion » : c'est ce qu'il écrit au philosophe Christoph Schrempf ${ }^{49}$. Le Voyage en Orient constitue précisément le récit, à la fois autobiographique et universel, de cette entreprise insensée, aberrante (töricht) et impossible qu'est l'écriture poétique. Dans cette allégorie se plaçant sous le signe du Roland furieux ${ }^{50}$, Hesse prend la suite du grand « historiographe de l'Ordre » qu'est l'Arioste, lequel déjà décrivait, malgré les «moqueries de la foule bornée » et

47. - Ibid., « Für Marulla » (1953), p. 932 : « ... auf der Nürnberger Reise, [...] in einer krisenhaften und oft bösen Zeit meines Lebens, der die Katharsis durch den Steppenwolf noch nicht gelungen war».

48. - H. Hesse, Materialien zum Steppenwolf, op. cit., Lettre du 5.8.1926, p. 95.

49. - H. Hesse, Gesammelte Briefe, op. cit., "Lettre de l'été 1932 à Christoph Schrempf », p. 341.

50. - Cf. Marc Fumaroli, « Allégorie et ironie au XVIII siècle : Fragonard et ses dessins pour le Roland Furieux », Revue d'histoire littéraire de France, Paris, Presses Universitaires de France, 2012/2, vol. 112, p. 443-477. 
le « peu de créance que l'inexpérience accordera à son chant », un « voyage » où il « vit des choses très éloignées de ce qu'il tenait pour vérité » 51 : tels une enveloppe sphérique irisée autour d'un vide mutique, les premier et dernier chapitres décrivant l'Ordre, ses membres et le Voyage « convoquent » de fait « sur un mode créateur (schöpferisch) le passé, l'avenir et l'imaginaire (das Gewesene, das Zukünftige, das Erdichtete) dans le moment présent », lors même que les trois chapitres centraux évoquent l'échec de la narration, le chaos, l'absence de sens dont désespère le narrateur. Celui-ci verra, dans les Archives de l'Ordre, son récit se dissoudre sous ses yeux en allégories, « en traits, en points, en cercles », en « ornements agréables dénués de toute signification » (ML78).

L'impossibilité de l'entreprise de narration repérée par Günter Eich s'avère un leitmotiv traversant tous les chapitres du récit d'un voyage comme échec narratif. Elle constitue le noyau de la poétique hesséenne de l'allégresse, le fondement humoristique de son geste d'écriture, si l'on en croit la définition, d'origine chinoise, qu'il propose de l'humour. Ce dernier caractérise en effet l'attitude de Confucius selon les sages chinois : «N'est-ce pas celui qui, sachant que c'est impossible, le fait quand même ? ${ }^{52}$. Un tel humour se retrouve chez tout narrateur d'histoire, qu'il soit écrivain ou historien, écrit Hesse en 1933 à Thomas Mann :

... au milieu de la conception actuelle de l'histoire et de son écriture m'est devenue infiniment chère l'ironie silencieuse, teintée de mélancolie, avec laquelle vous considérez en définitive la problématique de toute histoire et de toute volonté de narration sans pour autant faiblir un instant dans votre effort pour cette écriture d'histoire reconnue comme dans le fond impossible. Cela précisément m'est [...] profondément sympathique et familier : entreprendre l'impossible, alors qu'on en est averti, prendre activement sur soi le tragique ${ }^{53}$.

Si Hesse emploie ici le terme d'ironie, peut-être à la fois pour user d'un terme cher à Thomas Mann et pour qualifier une tonalité mannienne plus mélancolique, différant de la sienne, alors plus féerique, plus lumineuse, il n'omet pas de souligner la dimension tragique du geste d'écriture. L'humour dont il est question fait un avec la vertu, tragique, d'allégresse qui sera thématisée dans Le Jeu des perles de verre pour qualifier l'atti-

51. - H. Hesse cite ces vers de l'Arioste dans les premières pages de son récit (ML11).

52. - H. Hesse, « Lieblingslektüre » (1945), Gesammelte Schriften VII, op. cit., p. 420 : « Kung Fu Tse wird gelegentlich so charakterisiert : "Ist das nicht der, der weiß, daß es nicht geht, und es dennoch tut? " Das ist von einer Gelassenheit, einem Humor und einer Schlichtheit, für die ich in keiner Literatur ein ähnliches Beispiel weiß».

53. - Idem, « Brief von Ende 1933 an Thomas Mann», p. 561. 
tude de Josef Knecht comme de toute écriture artistique, existence et art se fondant, sous deux modes d'objectivations de l'esprit, en une seule et même manière pour l'homme d'être au monde, de devenir homme. Cet humour, très éloigné de tout humour conciliateur et " doré ${ }^{54}$ bourgeois, relève d'un « humour du pendu » (Galgenhumor) ${ }^{55}$ lucide, sans espoir de salut, faisant face à la mort, à l'inexistence du sujet comme à la vacuité de la langue, système de conventions allégoriques ${ }^{56}$ dont aucun sens réel ne saurait émerger, dans lequel aucune compréhension, aucun dialogue réels ne sauraient advenir ${ }^{57}$ : l'impossibilité narrative est consubstantielle au sujet et à la langue et se fonde dans une double inconsistance ontologiquement une.

Ce constat ressort de l'expérience qui, au cœur du Voyage en Orient, constitue le noyau, entre vide et chaos, de l'écriture poétique et historique selon Hesse et présente de nombreux points communs avec l'expérience de Lord Chandos dans «Une lettre » de Hofmannsthal, autre récit jouant entre histoire et autobiographie. Nous l'évoquerons en guise de conclusion.

\section{Conclusion}

Dans Le Voyage en Orient, H. H. réalise en effet qu'à peine commencé, son récit «se perd dans le vague et l'incompréhensible». Tous ces événements dont il aimerait conter «le déroulement»se délitent sous son « examen attentif», « tout fuit et se dissout », « nulle part » « une unité imaginaire », « une idée », « un centre, un point autour duquel tourne la roue » (ML 45). Aucune quête de vérité, aucune véracité ne sont plus possibles, l'oubli gangrène l'esprit du plus consciencieux des historiens (ML100). Le doute lié à la perte de la foi dans la puissance signifiante du langage contamine la mémoire et va jusqu'à remettre en question la réalité de l'expérience vécue (ML45). Le sujet perd toute substance :

54. - H. Hesse, Gesammelte Briefe, op. cit., « Lettre à Alfred Kubin, fin mars $1932 »$, p. 329.

55. - Hermann Hesse, in Volker Michels, Materialien zum Glasperlenspiel, vol. 1, Frankfurt a.M., Suhrkamp, 1973, « Brief, März 1938, an Alfred Kubin », p. 184: « ... in unsern schwächern, aber auch differenzierteren Seelen gedeiht [...] der Humor, der echte, nicht der goldene, sondern eben der Galgen- und Narrenhumor ».

56. - H. Hesse, « Geheimnisse », Gesammelte Schriften, op. cit., p. 790 : «In dieser erträglichen, lauen, geordneten Zone der Begriffe, der Systeme, der Dogmatiken, der Allegorien leben wir neun Zehntel unsres Lebens ».

57. - Ibid. : « ...auch [der Dichter] [...] wendet sich mit dem, was er schreibt, an seine Leser meistens durchaus mit der wohlgepflegten Illusion, es bestehe eine Norm, eine Sprache, ein System, das es ihm ermögliche, seine Gedanken und Erlebnisse so mitzuteilen, daß der Leser sie gewissermaßen miterleben und sich tatsächlich aneignen könne ». 
Tout n'est que masse informe d'images qui se sont reflétées dans quelque chose, et ce quelque chose est mon propre moi, et ce moi, ce miroir, où que je l'interroge, n'est rien d'autre que néant, que l'enveloppe supérieure d'une surface de verre $(\mathrm{ML} \mathrm{45})^{58}$.

Chandos évoque de son côté l'impossibilité d'une pensée ou d'un discours suivis devant la « décomposition » du langage en «fragments » qui « ne se [laissent] plus enfermer dans un concept», en mots-« tourbillons » «à travers » lesquels « on atteint le vide » lorsqu'on y plonge le regard ${ }^{59}$. Une même défiance nietzschéenne face au langage et aux mots barrant l'accès aux choses, un rejet analogue de tout volontarisme et Führertum à la Stefan George sont chez Hofmannsthal ${ }^{60}$ comme chez Hesse $^{61}$ à l'origine de cette expérience à la fois ontologiquement dévastatrice et poétiquement fondatrice où la langue s'avère système abstrait de conventions allégoriques dont s'absente toute signification réelle, qui n'autorise aucune communication réelle et où « la mémoire culturelle et scientifique » se perd en «se transformant en chaos ${ }^{62}$.

Les conséquences poétiques qu'en tirent les deux auteurs semblent toutefois différer. Certes, dans les deux cas, « le narrateur doit mourir à lui-même pour que son langage renaisse », pour qu'il redevienne « voyant ${ }^{63}$ et puisse vivre une nouvelle expérience, à la fois aimante et tragique, de pleine «présence » (Gegenwart), d'heureuse harmonie renouvelée avec le monde ${ }^{64}$. Dans les deux cas, une certaine passivité en est reconnue le préalable nécessaire. Seulement, chez ChandosHofmannsthal, « l'attitude d'attente silencieuse et d'écoute » 65 s'opère vis-à-vis des petites choses, un arrosoir, un chien. La « révélation » ou épiphanie, le « flux soudain de sentiment divin » (jäh steigende Flut

58. - La fin de la traduction officielle n'a pu être retenue, « oberste Haut einer Glasfläche » ayant été traduit par Jean Lambert par « la surface d'un flacon ».

59. - Hugo von Hofmannsthal, «Ein Brief », Gesammelte Werke. Erzählungen, p. 461-472. Ici p. 466 : «Es zerfiel mir alles in Teile, die Teile wieder in Teile und nichts mehr ließ sich mit einem Begriff umspannen. Die einzelnen Worte schwammen um mich [...] Wirbel sind sie, [...] durch die hindurch man ins Leere kommt».

60. - Cf. Jacques Le Rider, « La "Lettre de Lord Chandos" » in : Littérature, n 95, 1994, p. 93-110. Ici, p. 108.

61. - H. Hesse, Gesammelte Schriften, op. cit., «Brief an Kuno Fiedler, Januar 1940 », p. 617 : « ...ich habe weder schädliche Lehren und Haltungen in die Welt gebracht, wie ein Nietzsche oder auch ein Stefan George, noch habe ich aus der Nachahmung solcher Haltungen einen Kult gemacht ».

62. - Cf. J. Le Rider, "La "Lettre de Lord Chandos" », op. cit., p. 107.

63. - Ibid., p. 97.

64. - H. v. Hofmannsthal, «Ein Brief », op. cit., p. 469: « ... es war Gegenwart, die vollste erhabenste Gegenwart... », « ...diese mich und die ganze Welt durchwebende Harmonie... ».

65. - J. Le Rider, « La "Lettre de Lord Chandos" », op. cit., p. 107. 
göttlichen Gefühls) ${ }^{66}$ advient sur le plan de la corporéité67, à l'écart de l'esprit et reste réfractaire au langage.

Chez Hesse au contraire, la passivité de la souffrance, d'un Erdulden, la "persévérance dans le chaos », devient active, se fait écriture poétique. Elle mène en effet à la découverte d'un nouvel art d'écrire qui, allègre, assume l'allégorie et l'incompréhension comme condition préalable 68 à une « parole pleine ${ }^{69}$, à un dialogue authentique au sein d'une réalité culturelle, d'une communauté historique et atemporelle de l'esprit qui, de la sorte, perdurent. Le Voyage en Orient en offre le témoignage par le récit tout entier qui rend manifeste la possibilité d'une réintégration de l'ordre à l'issue d'une lettre rédigée sans maîtrise, sous la fièvre (ML70), autre récit allégorique fonctionnant comme appel, certes illisible et « aberrant » (töricht), mais recevant tout de même une réponse. Une lecture de vérité s'avère allégoricienne lorsqu'elle est authentique, à l'image de celle que réalisent les « Supérieurs de l'Ordre » et « Serviteurs » de l'esprit et de la culture. Toute lecture, préalable à toute réponse et au dialogue qu'est toute culture entre appel et réponse, retrouve le chemin menant de la « rune » à l'« appel », au « battement de cœur » premier dont vit l'homme, dans la langue et l'esprit ${ }^{70}$. L'écrit fondamental et originaire que représente la « lettre de l'Ordre » (Bundesbrief), où se donne à lire «le secret » de l'Ordre, en apporte une seconde preuve. Si sa perte est bien à l'origine, chez $\mathrm{H}$. H., de la perte de foi dans l'existence même de l'Ordre, donc de sa perte de foi dans le langage, et si cette « lettre » lui reste incompréhensible, car écrite dans la langue originelle et secrète des « adeptes » (ML79), lorsque H. H. la retrouve dans les archives de l'Ordre, elle n'en demeure pas moins trace, « hiéroglyphe » témoignant de l'existence atemporelle de l'Ordre : si l'écriture poétique n'est plus un « dévoilement de vérité » (Entbergen der

66. - H. v. Hofmannsthal, « Ein Brief », op. cit., p. 467.

67. - Ibid., p. 469 : «Es ist mir dann, als bestünde mein Körper aus lauter Chiffren, die mir alles aufschließen ».

68. - H. Hesse, Gesammelte Briefe, op. cit., « Lettre à Hilde Saenger, 1931 », p. 305 : « ... lernen Sie das Nichtverstehen, die Sinnlosigkeit als Vorbedingung für alles erkennen, was der Mensch wert sein kann. Wie Sie nachher Ihren Glauben formulieren, ob christlich oder sonstwie ist einerlei ».

69. - H. Hesse, Gesammelte Schriften VII, op. cit., «Rundbrief aus Sils Maria», p. 921. Hesse cite Martin Buber : « Der Krieg hat von je einen Widerpart, der fast nie als solcher hervortritt, aber in der Stille sein Werk tut : die Sprache - die erfüllte Sprache, die Sprache des echten Gesprächs, in der Menschen einander verstehen und sich miteinander verständigen ».

70. - Cf. H. Hesse, "Rückblick », fragment (1937), in Materialien zum Glasperlenspiel, op. cit., p. 383 : « Nun ihr Freunde [...] / ihr findet in meinem bedächtig Geschriebnen /Das lebendige Wort, das liebende, / Wisset die Rune zum Ruf / Fern in Nah, und Gedichte / Rückzuverwandeln in Herzschlag... ». 
Wahrheit ${ }^{71}$ affirmatif, elle s'accomplit dans la dissimulation protectrice (Bergen et Verbergen) d'une indicible vérité, là même où elle entreprend l'impossible « représentation de l'irreprésentable » déplorée par Günter Eich. Avant de s'offrir en sacrifice à cette écriture dédiée à l'esprit et à la vérité, $\mathrm{H}$. $\mathrm{H}$. a ainsi l'intuition, face à tous les récits et variantes infinis du «Voyage » qu'il découvre dans les Archives, que, si «tout se déplace, se transforme, se distord dans tous ces miroirs », c'est « le visage de la vérité » lui-même qui, avec « ironie », toujours " inaccessible », s'y donne à lire « en se cachant derrière tous ces rapports, ces contre-rapports et ces légendes » (ML 100).

Par un jeu de traces allégoriques à fonction d'appel, dont la fascination est d'autant plus bénéfique qu'elle se réfléchit thématiquement et se défait sous le regard critique d'un auteur-lecteur allégoricien, Hermann Hesse entraîne dans Le Voyage en Orient le lecteur à vivre la langue dans son essence, à vivre dans la langue, par essence dialogue, fondamentalement parole et écoute, l'expérience concrète d'une surréalité culturelle qui, allègre, le dote à la fois d'allégresse et de la vertu humaine d'allégresse qu'est l'humour, c'est-à-dire à la fois des forces et du recul nécessaires pour ne céder ni aux leurres ni aux influences néfastes de tout ce qui l'éloigne de son esprit et de son humanité mais pour leur opposer au contraire sa propre interprétation du monde. Tel est en tous les cas, selon ses propres dires, le propos poétique et politique de son manifeste :

L'atmosphère du Voyage en Orient et de l'Ordre, cette vie au sein de l'univers atemporel de l'esprit, au sein d'Idées et de représentations de nombreuses époques et cultures [...], cette faculté de vivre dans un royaume atemporel n'a en soi rien d'une faiblesse à mes yeux, mais m'apparaît bien plus comme une force, comme la seule force peut-être de l'homme actuel. Le manque qui est le nôtre en raison de l'absence d'une culture encore ascendante et en fleurs nous est en partie remplacé par la possibilité de faire, par-delà les cultures, de l'humanité, par-delà le présent, de l'éternel notre oxygène, l'air à respirer pour vivre. De ce monde atemporel des religions, des philosophies et des arts, on revient aux problèmes actuels, même pratiques et politiques, non affaibli mais fortement affermi, armé de patience, d'humour, d'une volonté nouvelle de comprendre, d'un amour renouvelé pour le vivant, pour ses urgences et ses errances ${ }^{72}$.

71. - J. Söring, « Die Grenze der Darstellbarkeit », op. cit., p. 514.

72. - H. Hesse, « Suchen nach Gemeinschaft» (1932) in : Schriften zur Literatur I, op. cit., p. 88. 
